\section{Fatores associados à hipotensão ortostática em adultos: estudo ELSA-Brasil}

\author{
Factors associated with orthostatic hypotension \\ in adults: the ELSA-Brasil study
}

\section{Factores asociados a la hipotensión ortostática en adultos: estudio ELSA-Brasil}

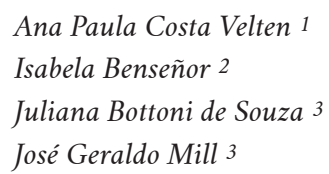

doi: 10.1590/0102-311X00123718

\section{Resumo}

O objetivo foi investigar os fatores associados à presença de hipotensão ortostática em 14.833 indivíduos de 35-74 anos. Estudo transversal realizado com os dados da linha de base (2008-2010) do Estudo Longitudinal de Saúde do Adulto (ELSA-Brasil). O teste postural foi realizado após repouso de 20 minutos na posição supina e adoção ativa da postura ortostática. A pressão arterial foi medida em supino e aos três minutos de ortostase com aparelho oscilométrico (HEM 705 CP, Omron, São Paulo, Brasil). A hipotensão ortostática foi definida por queda $\geq 20 \mathrm{mmHg}$ na pressão arterial sistólica el ou queda $\geq 10 \mathrm{mmHg}$ na pressão arterial diastólica. As covariáveis analisadas foram sexo, faixa etária, raça/cor, escolaridade, estado nutricional, circunferência da cintura, alteração no índice tornozelo braquial, velocidade de onda de pulso, pressão arterial sistólica e diastólica, hipertensão, diabetes, uso de anti-hipertensivos, colesterol, triglicérides, sorologia para a doença de Chagas, ocorrência de sintomas e variação de frequência cardíaca no teste postural, relato de doença cardiaca, infarto agudo do miocárdio (IAM)/revascularização e acidente vascular cerebral. A hipotensão ortostática foi significativamente associada à maior faixa etária, $O R=1,83$ (IC95\%: 1,14-2,95); alteração no índice tornozelo braquial, $O R=2,8$ (IC95\%: 1,13-6,88), IAM/revascularização, $O R=1,70$ (IC95\%: 1,01-2,87); relato de doença cardíaca, OR = 3,03 (IC95\%: 1,71-5,36); pressão arterial sistólica aumentada, OR = 1,012 (IC95\%: 1,0061,019); sorologia positiva para a doença de Chagas, OR = 2,29 (IC95\%: 1,234,27) e ocorrência de sintomas na mudança postural, OR = 20,81 (IC95\%: 14,81-29,24). A presença de hipotensão ortostática pode ser alerta de potencial comprometimento cardiovascular, e, portanto, uma ferramenta de rastreamento e prevenção.

Hipotensão Ortostática; Doenças Cardiovasculares; Saúde do Adulto
Correspondência

A. P. C. Velten

Universidade Federal do Espírito Santo.

Rod. BR 101 Norte, Km 60, São Mateus, ES 29932-540, Brasil. paulinhavelten@hotmail.com

1 Universidade Federal do Espírito Santo, São Mateus, Brasil. 2 Universidade de São Paulo, São Paulo, Brasil.

3 Universidade Federal do Espírito Santo, Vitória, Brasil. 


\section{Introdução}

A hipotensão ortostática é definida como a redução sustentada de, pelo menos, $20 \mathrm{mmHg}$ da pressão arterial sistólica (PAS) e/ou de $10 \mathrm{mmHg}$ da pressão arterial diastólica (PAD) dentro de três minutos após a adoção da ortostase ${ }^{1}$. A etiologia da hipotensão ortostática é diversa, mas é sempre ocasionada por falha nos mecanismos neurais e/ou circulatórios de compensação da redução do retorno venoso e, consequentemente, do débito sistólico e da pressão arterial. A queda pressórica diminui o fluxo sanguíneo cerebral e pode provocar sintomas como tontura, náusea, alterações visuais e até síncope. Entretanto, a hipotensão ortostática, geralmente, é assintomática 2.

A hipotensão ortostática tem relação com a disfunção autonômica primária (como a insuficiência autonômica pura e a insuficiência autonômica na doença de Parkinson) ou secundária (como a causada por polineuropatias associadas a diabetes, fármacos, doenças autoimunes), além de outros fatores não neurogênicos, como a hipovolemia, função cardíaca deprimida, uso de medicamentos e idade avançada 3. Em estudos de populações representativas da população geral, a hipotensão ortostática tem sido associada a comorbidades cardiovasculares, como doença coronariana 4, fibrilação atrial 5,6, hipertensão 7, insuficiência cardíaca 8,9,10, acidente vascular cerebral (AVC) 11,12, aumento da rigidez arterial 13; doença renal crônica 14, além de debilidade no estado geral de saúde 15,16. A presença de hipotensão ortostática também se associa diretamente com a mortalidade. Destaca-se, entretanto, que há estudos que não detectam associação com algumas dessas condições, ou até associações negativas 17,18,19, o que justifica a necessidade de estudos adicionais nessa área, notadamente pelo envelhecimento progressivo da população, haja vista a associação constante entre a hipotensão ortostática e a idade.

A prevalência de hipotensão ortostática na população geral é muito diversa em diferentes estudos, indo desde 2,73\%, no estudo de Rose et al. 7, até 58,6\%, no de Cooke et al. ${ }^{20}$. A maioria, entretanto, situa-se entre 5\% em populações mais jovens (45-64 anos) 21 até cerca de 30\% em populações de idosos (> 65 anos) 22. Essa variação reflete não só a idade, mas também a variedade de métodos utilizados na definição e averiguação da hipotensão ortostática.

Os estudos de associação realizados até o momento foram conduzidos em populações da América do Norte, Europa e Ásia, não havendo estudos dessa natureza na população brasileira. Diante do exposto, este trabalho tem como objetivo investigar quais fatores se associam à presença de hipotensão ortostática em uma grande amostra brasileira.

\section{Material e métodos}

Estudo transversal realizado com os dados da linha de base (2008-2010) do Estudo Longitudinal de Saúde do Adulto (ELSA-Brasil). O ELSA-Brasil é uma coorte de 15.105 servidores públicos, ativos e aposentados, de ambos os sexos e com idade entre 35-74 anos. Desses, 14.833 (98,2\%) possuem dados completos da manobra postural realizada para identificar a presença de hipotensão ortostática. O ELSA-Brasil tem como objetivo principal determinar a incidência de doenças crônicas e os seus determinantes proximais e distais. A pesquisa vem sendo conduzida em seis centros de investigação localizados nas regiões Nordeste, Sudeste e Sul do país; estando cinco sediados em instituições públicas de ensino superior - Universidade Federal da Bahia (UFBA), Universidade Federal do Espírito Santo (UFES), Universidade Federal de Minas Gerais (UFMG), Universidade Federal do Rio Grande do Sul (UFRGS) e Universidade de São Paulo (USP) - e um em uma instituição de pesquisa do Ministério da Saúde, a Fundação Oswaldo Cruz (Fiocruz), no Rio de Janeiro . Os participantes são servidores, ativos ou aposentados dessas instituições, recrutados como voluntários de pesquisa. Detalhes sobre amostragem, recrutamento e dados coletados na linha de base foram publicados anteriormente 23,24.

A prevalência de hipotensão ortostática na linha de base do estudo foi de $2,02 \%$, com frequência absoluta de 300 participantes. Para classificar o participante como portador de hipotensão ortostática, utilizou-se a definição de queda da PAS $\geq 20 \mathrm{mmHg}$ e/ou da $\mathrm{PAD} \geq 10 \mathrm{mmHg}$ na medida pressórica obtida três minutos após adoção ativa da ortostase 1,25 .

Para a realização da manobra postural, o participante permanecia deitado por cerca de 20 minutos, enquanto era submetido à aferição do índice tornozelo-braquial (ITB). Foram obtidas três medidas 
da pressão arterial no braço direito na posição supina com intervalo de dois minutos entre elas. Utilizou-se a média das duas últimas medidas como o valor da pressão arterial em supino. Em seguida, o aferidor instruía o participante a se levantar (se necessário com ajuda) e a manter postura ereta, com apoio no piso apenas dos pés. A pressão arterial era novamente medida aos dois, três e cinco minutos após adoção da ortostase 26.

Em formulário próprio, o aferidor deveria anotar a presença de sintomas relatados espontaneamente (tontura, alterações visuais, náusea etc.). Em função da intensidade dos sintomas, o protocolo podia ser alterado, fazendo-se as medidas da pressão arterrial na posição sentada. As medidas da pressão arterial foram obtidas com aparelho oscilométrico (HEM 705 CP, Omron, São Paulo, Brasil) com tamanho do manguito de acordo com a circunferência do braço. Em 27 participantes, foi necessária a utilização do esfigmomanômetro de mercúrio (Unitec Hospitalar, São Paulo, Brasil) por falha na leitura do aparelho oscilométrico. Outros 14 participantes não conseguiram manter a ortostase para todas as medidas e tiveram que retornar à posição supina com aumento da pressão arterial. Para esses, fez-se uma correção baseada na média dos deltas (diferença da pressão arterial medida em pé e a supina) da pressão arterial dos indivíduos que permaneceram eretos com o mesmo valor de queda pressórica.

As covariáveis incluídas na análise foram: sexo, faixa etária (35-44 anos, 45-54 anos, 55-64 anos e 65-74 anos), raça/cor autodeclarada (branca, preta, parda, amarela ou indígena), escolaridade, estado nutricional avaliado pelo índice de massa corporal (IMC), circunferência da cintura (CC), alteração no ITB, velocidade de onda de pulso (VOP), autorrelato de diagnóstico médico prévio de doença cardíaca, infarto agudo do miocárdio (IAM) ou revascularização (cirurgia cardíaca ou angioplastia ou colocação de stent), AVC, presença de diabetes mellitus, hipertensão arterial, uso de anti-hipertensivo, pressão arterial sistólica e diastólica, colesterol total, LDL-c e HDL-c, triglicérides, sorologia positiva para doença de Chagas (ELISA), presença de sintomas no teste postural, variação da frequência cardíaca (FC) após a mudança postural, tabagismo, uso de álcool e atividade física no lazer.

A alteração do ITB foi definida pela razão entre a média de duas verificações da pressão arterial sistólica tibial, (direita e/ou esquerda), e a maior pressão arterial sistólica no braço direito menor que 0,9 26. A VOP foi obtida medindo-se, simultaneamente, os pulsos nas artérias carótida direita e femoral direitas (Complior, Artech Medicale, França) e a distância entre a fúrcula esternal e o pulso femoral (fita métrica). A VOP é dada como variável contínua pela divisão da distância fúrcula-femoral pelo intervalo de tempo entre os dois pulsos 26. Presença de hipertensão arterial foi definida pela ocorrência de $\mathrm{PAS} / \mathrm{PAD} \geq 140 / 90 \mathrm{mmHg}$ e/ou uso de medicação anti-hipertensiva, incluindo diuréticos. Para diabetes, pelo menos, um dos seguintes critérios deveria ser atendido: diabetes autorreferida, glicemia em jejum $\geq 126 \mathrm{mg} / \mathrm{dL}$, glicemia pós sobrecarga de glicose $\geq 200 \mathrm{mg} / \mathrm{dL}$, hemoglobina glicada $\geq 6,5 \%$ ou uso de antidiabético oral e/ou insulina. A variação da FC consistiu na diferença (delta) entre a FC em supino e após três minutos em ortostase. Atividade física no lazer foi classificada em fraca (< $600 \mathrm{MET}$-minutos/semana), moderada (600 a 1.500 MET-minutos/semana) ou forte ( $>1.500 \mathrm{MET}$ minutos/semana), de acordo com o International Physical Activity Questionnaire - IPAC, versão longa 27.

Os dados dessas variáveis foram obtidos em entrevista com aplicação de questionários padronizados e validados ou pela realização de exames clínicos e laboratoriais. Os exames foram realizados em ambiente silencioso, com temperatura entre 20 e $24^{\circ} \mathrm{C}$, em um único dia e no período matutino. Os dados foram coletados por aferidores previamente treinados e certificados. Detalhes dos procedimentos adotados estão disponíveis 26 , assim como rotinas de treinamento e certificação dos aferidores e entrevistadores 28 .

Os dados foram, inicialmente, submetidos à análise univariada, sendo utilizados o teste de hipóteses qui-quadrado e o teste $U$ de Mann-Whitney. As associações foram verificadas por meio de regressão logística testada para as variáveis significantes na análise univariada, com cálculo de odds ratio (OR) e intervalo de 95\% de confiança (IC95\%), com o método forward stepwise (condicional). Realizou-se também análise por subgrupos de faixa etária (menor que 44 anos, entre 45-59 anos e maior que 60 anos). Nos subgrupos de faixa etária, em todos os testes, o nível de significância foi de $\mathrm{p}<0,05$. As análises foram efetuadas com o programa IBM SPSS Statistics 21 (https://www.ibm.com/).

O ELSA-Brasil foi submetido e aprovado nos Comitês de Ética em Pesquisa das instituições envolvidas, e todos os participantes assinaram o termo de consentimento 29 . 


\section{Resultados}

De acordo com os dados deste estudo, faixa etária mais alta, alteração no ITB, PAS mais alta e presença prévia de IAM/revascularização, doença cardíaca, sorologia positiva para a doença de Chagas e, principalmente, ocorrência de sintomas na mudança postural foram significativamente associadas à presença de hipotensão ortostática. Ao subdividir a amostra em diferentes faixas etárias, a sorologia positiva para a doença de Chagas e a ocorrência de sintomas na mudança postural foram fatores associados nos indivíduos com menos de 44 anos; hipertensão, maior VOP e a presença de sintomas, nos indivíduos de 45-59 anos; e, nos com mais de 60, esses fatores foram menor escolaridade, IAM ou revascularização, doença cardíaca e, mais uma vez, a presença de sintomas durante a mudança da postura.

A Tabela 1 apresenta características gerais dos indivíduos, incluindo variáveis sociodemográficas e clínicas em função da presença de hipotensão ortostática. Observa-se que a frequência de hipotensão ortostática é maior nos indivíduos mais velhos e com menor escolaridade. A hipotensão ortostática também foi mais frequente nos indivíduos com ITB alterado e em presença de todas as comorbidades listadas (exceto AVC). O relato de tabagismo, consumo de álcool ou prática de atividade física não influenciou no aparecimento de hipotensão ortostática. A Tabela 2 mostra que os portadores de hipotensão ortostática também apresentaram mediana mais alta de VOP e PAS e menor aumento de FC após a manobra postural.

Os resultados da regressão logística, expressos pela razão de chances, são mostrados na Tabela 3. Apesar de significativa no nível de 5\%, a presença de hipertensão não foi incluída no modelo por colinearidade com a PAS, assim como a VOP. O modelo final incluiu as variáveis faixa etária, alteração no ITB, IAM/revascularização, doença cardíaca, PAS, sorologia para a doença de Chagas e presença de sintomas após a mudança postural; todas associadas significativamente à hipotensão ortostática.

Tabela 1

Características sociodemográficas, dados clínicos, história médica pregressa e hábitos de vida, análise univariada (variáveis qualitativas). Estudo Longitudinal de Saúde do Adulto (ELSA-Brasil, 2008-2010).

\begin{tabular}{|c|c|c|c|c|c|}
\hline \multirow[t]{3}{*}{ Variáveis } & \multicolumn{4}{|c|}{ Hipotensão ortostática } & \multirow[t]{3}{*}{ Valor de $p$} \\
\hline & \multicolumn{2}{|c|}{ Sim } & \multicolumn{2}{|c|}{ Não } & \\
\hline & $\mathbf{n}$ & $\%$ & $\mathbf{n}$ & $\%$ & \\
\hline \multicolumn{6}{|l|}{ Características sociodemográficas } \\
\hline \multicolumn{6}{|l|}{ Faixa etária (anos) } \\
\hline $35-44$ & 39 & 1,2 & 3.259 & 98,8 & $<0,000 *$ \\
\hline $45-54$ & 93 & 1,6 & 5.732 & 98,4 & \\
\hline $55-64$ & 116 & 2,8 & 4.041 & 97,2 & \\
\hline $65-74$ & 52 & 3,3 & 1.501 & 96,7 & \\
\hline \multicolumn{6}{|l|}{ Sexo } \\
\hline Masculino & 135 & 2,0 & 6.661 & 98,0 & 0,774 \\
\hline Feminino & 165 & 2,1 & 7.872 & 97,9 & \\
\hline \multicolumn{6}{|l|}{ Cor ou raça } \\
\hline Preta & 59 & 2,5 & 2.283 & 97,5 & 0,153 \\
\hline Parda & 81 & 2,0 & 4.029 & 98,0 & \\
\hline Branca & 139 & 1,8 & 7.540 & 98,2 & \\
\hline Amarela/Indígena & 13 & 2,5 & 512 & 97,5 & \\
\hline \multicolumn{6}{|l|}{ Escolaridade } \\
\hline Ensino Fundamental completo & 57 & 3,0 & 1.826 & 97,0 & 0,002 * \\
\hline Ensino Médio completo & 110 & 2,1 & 5.023 & 97,9 & \\
\hline Ensino Superior & 133 & 1,7 & 7.684 & 98,3 & \\
\hline
\end{tabular}


Tabela 1 (continuação)

\begin{tabular}{|c|c|c|c|c|c|}
\hline \multirow[t]{3}{*}{ Variáveis } & \multicolumn{4}{|c|}{ Hipotensão ortostática } & \multirow[t]{3}{*}{ Valor de $p$} \\
\hline & \multicolumn{2}{|c|}{ Sim } & \multicolumn{2}{|c|}{ Não } & \\
\hline & $\mathbf{n}$ & $\%$ & $\mathbf{n}$ & $\%$ & \\
\hline \multicolumn{6}{|c|}{ Estado clínico e história médica pregressa } \\
\hline \multicolumn{6}{|l|}{ Estado nutricional } \\
\hline Magreza & 2 & 1,4 & 139 & 98,6 & 0,407 \\
\hline Eutrofia & 114 & 2,1 & 5.244 & 97,9 & \\
\hline Sobrepeso & 128 & 2,1 & 5.871 & 97,9 & \\
\hline Obeso & 56 & 1,7 & 3.277 & 98,3 & \\
\hline \multicolumn{6}{|l|}{ Alteração no ITB } \\
\hline Não & 291 & 2,0 & 14.427 & 98,0 & $<0,000$ * \\
\hline Sim & 6 & 8,5 & 65 & 91,5 & \\
\hline \multicolumn{6}{|l|}{ IAM/Revascuilarização } \\
\hline Não & 277 & 1,9 & 14.163 & 98,1 & $<0,000$ * \\
\hline Sim & 21 & 5,5 & 363 & 94,5 & \\
\hline \multicolumn{6}{|l|}{ Doença cardíaca } \\
\hline Não & 283 & 1,9 & 14.304 & 98,1 & $<0,000$ * \\
\hline Sim & 17 & 7,3 & 215 & 92,7 & \\
\hline \multicolumn{6}{|l|}{ AVC } \\
\hline Não & 297 & 2,0 & 14.350 & 98,0 & 0,711 \\
\hline $\operatorname{Sim}$ & 3 & 1,6 & 180 & 98,4 & \\
\hline \multicolumn{6}{|l|}{ Diabetes } \\
\hline Não & 224 & 1,9 & 11.712 & 98,1 & 0,010 * \\
\hline Sim & 76 & 2,6 & 2.818 & 97,4 & \\
\hline \multicolumn{6}{|l|}{ Hipertensão } \\
\hline Não & 154 & 1,6 & 9.411 & 98,4 & $<0,000$ * \\
\hline Sim & 146 & 2,8 & 5.109 & 97,2 & \\
\hline \multicolumn{6}{|l|}{ Uso de anti-hipertensivo } \\
\hline Não & 178 & 1,7 & 10.354 & 98,3 & $<0,000$ * \\
\hline Sim & 121 & 2,8 & 4.162 & 97,2 & \\
\hline \multicolumn{6}{|c|}{ Sorologia para a doença de Chagas positiva } \\
\hline Não & 288 & 2,0 & 14.222 & 98,0 & 0,008 * \\
\hline Sim & 12 & 4,2 & 272 & 95,8 & \\
\hline \multicolumn{6}{|c|}{ Sintomas após mudança postural } \\
\hline Não & 241 & 1,7 & 14.332 & 98,3 & $0,000 *$ \\
\hline Sim & 59 & 22,7 & 201 & 77,3 & \\
\hline \multicolumn{6}{|l|}{ Hábitos de vida } \\
\hline \multicolumn{6}{|l|}{ Tabagismo } \\
\hline Não & 164 & 1,9 & 8.277 & 98,1 & 0,428 \\
\hline Sim & 136 & 2,1 & 6.255 & 97,9 & \\
\hline \multicolumn{6}{|l|}{ Uso de álcool } \\
\hline Nunca & 37 & 2,3 & 1.540 & 97,7 & 0,290 \\
\hline Ex-usuário & 67 & 2,3 & 2.898 & 97,7 & \\
\hline Usuário & 195 & 1,9 & 10.075 & 98,1 & \\
\hline \multicolumn{6}{|l|}{ Atividade física no lazer } \\
\hline Fraca & 221 & 2,0 & 11.022 & 98,0 & 0,309 \\
\hline Moderada & 50 & 2,4 & 1.995 & 97,6 & \\
\hline Forte & 24 & 1,8 & 1.306 & 98,2 & \\
\hline Total de indivíduos (n) & & & & & \\
\hline
\end{tabular}

AVC: acidente vascular cerebral; IAM: infarto agudo do miocárdio; ITB: índice tornozelo braquial.

* Nível de significância menor que 5\%. 
Tabela 2

Dados clínicos e bioquímicos, análise univariada (variáveis quantitativas). Estudo Longitudinal de Saúde do Adulto (ELSA-Brasil, 2008-2010).

\begin{tabular}{|c|c|c|c|c|c|c|c|}
\hline \multirow[t]{3}{*}{ Variáveis } & \multicolumn{6}{|c|}{ Hipotensão ortostática } & \multirow[t]{3}{*}{ Valor de $p$} \\
\hline & \multicolumn{3}{|c|}{ Sim } & \multicolumn{3}{|c|}{ Não } & \\
\hline & Mediana & $\mathbf{P}_{25}$ & $\mathbf{P}_{75}$ & Mediana & $\mathbf{P}_{25}$ & $\mathbf{P}_{75}$ & \\
\hline Circunferência da cintura (cm) & 90,4 & 81,5 & 98,5 & 90,4 & 82,0 & 99,1 & 0,930 \\
\hline Velocidade de onda de pulso $(\mathrm{m} / \mathrm{s})$ & 9,5 & 8,4 & 11,3 & 9,0 & 8,0 & 10,1 & $<0,000 *$ \\
\hline Pressão arterial diastólica (mmHg) & 76 & 69 & 85 & 76 & 69 & 83 & 0,303 \\
\hline Pressão arterial sistólica (mmHg) & 124 & 112 & 138 & 119 & 109 & 131 & $<0,000 *$ \\
\hline Variação da frequência cardíaca (bpm) & 9,0 & 3,5 & 15,5 & 11,0 & 6,5 & 16,5 & $<0,000 *$ \\
\hline Colesterol total (mg/dL) & 215 & 186 & 242 & 211 & 186 & 239 & 0,601 \\
\hline Colesterol LDL (mg/dL) & 129 & 108 & 153 & 129 & 107 & 152 & 0,657 \\
\hline Colesterol HDL (mg/dL) & 55 & 46 & 66 & 54 & 46 & 65 & 0,439 \\
\hline Triglicérides (mg/dL) & 114 & 78 & 167 & 114 & 82 & 165 & 0,820 \\
\hline Total de indivíduos (n) & & 300 & & & 14.533 & & \\
\hline
\end{tabular}

* Nível de significância menor que 5\%.

\section{Tabela 3}

Variáveis associadas à presença de hipotensão ortostática. Estudo Longitudinal de Saúde do Adulto (ELSA-Brasil, 2008-2010).

\begin{tabular}{|c|c|c|c|c|c|c|}
\hline Variável & OR bruto & IC95\% & Valor de p & OR ajustado & IC95\% & Valor de $p$ \\
\hline \multicolumn{7}{|l|}{ Faixa etária (anos) } \\
\hline $35-44$ & 1,00 & & & 1,00 & & \\
\hline $45-54$ & 1,35 & $0,93-1,97$ & 0,113 & 1,22 & $0,81-1,82$ & 0,327 \\
\hline $55-64$ & 2,39 & $1,66-3,45$ & 0,000 & 2,02 & $1,36-3,02$ & 0,001 \\
\hline $65-74$ & 2,89 & $1,90-4,40$ & 0,000 & 1,83 & $1,14-2,95$ & 0,012 \\
\hline \multicolumn{7}{|l|}{ Escolaridade } \\
\hline Ensino Fundamental completo & 1,80 & $0,98-1,63$ & 0,000 & - & - & - \\
\hline Ensino Médio completo & 1,26 & $1,31-2,47$ & 0,071 & - & - & - \\
\hline Ensino Superior & 1,00 & & & & & \\
\hline \multicolumn{7}{|l|}{ Alteração no ITB } \\
\hline Não & 1,00 & & & 1,00 & & \\
\hline Sim & 4,57 & $1,96-10,64$ & & 2,80 & $1,13-6,88$ & 0,025 \\
\hline \multicolumn{7}{|l|}{ IAM/Revascularização } \\
\hline Não & 1,00 & & & 1,00 & & \\
\hline Sim & 2,95 & $1,87-4,66$ & 0,000 & 1,70 & $1,01-2,87$ & 0,044 \\
\hline \multicolumn{7}{|l|}{ Doença cardíaca } \\
\hline Não & 1,00 & & & 1,00 & & \\
\hline Sim & 3,99 & $2,40-6,64$ & 0,000 & 3,03 & $1,71-5,36$ & 0,000 \\
\hline \multicolumn{7}{|l|}{ Diabetes } \\
\hline Não & 1,00 & & & - & - & - \\
\hline $\operatorname{Sim}$ & 1,41 & $1,08-1,83$ & 0,011 & & & \\
\hline Pressão arterial sistólica (mmHg) & 1,02 & $1,011-1,023$ & 0,000 & 1,01 & $1,006-1,019$ & 0,000 \\
\hline \multicolumn{7}{|l|}{ Uso de anti-hipertensivo } \\
\hline Não & 1,00 & & & - & - & - \\
\hline Sim & 1,69 & $1,33-2,13$ & 0,000 & & & \\
\hline \multicolumn{7}{|l|}{ Sorologia para a doença de Chagas } \\
\hline Não & 1,00 & & & 1,00 & & \\
\hline $\operatorname{Sim}$ & 2,17 & $1,20-3,92$ & 0,010 & 2,29 & $1,23-4,27$ & 0,009 \\
\hline \multicolumn{7}{|l|}{ Sintomas na mudança postural } \\
\hline Não & 1,00 & & & 1,00 & & \\
\hline Sim & 17,45 & $12,71-23,96$ & 0,000 & 20,81 & $14,81-29,24$ & 0,000 \\
\hline Variação da frequência cardíaca (bpm) & 0,97 & $0,95-0,98$ & 0,000 & - & - & - \\
\hline
\end{tabular}

IAM: infarto agudo do miocárdio; IC95\%: intervalo de 95\% de confiança; ITB: índice tornozelo braquial; OR: odds ratio (razão de chances). 
A Tabela 4 apresenta os resultados das regressões logísticas, expressos pela razão de chances, para os subgrupos de faixa etária. A variável PAS, apesar de significativa na análise univariada de 45 a 59 anos, não foi incluída no modelo por colinearidade com hipertensão. As variáveis preenchidas, porém sem valor, foram incluídas por apresentarem significância na análise univariada, mas não permaneceram no modelo.

\section{Discussão}

Os achados deste estudo são congruentes com resultados de outras pesquisas, que associam a hipotensão ortostática ao aumento da idade 4,10,14,30, insuficiência cardíaca 8,9,10, doença coronariana 4,16,21, maior PAS e hipertensão 7,13,14,31,32, maior frequência de ITB alterado 15, maior rigidez arterial verificada por VOP 13,31 e menor escolaridade 12,15.

O desenho transversal adotado neste estudo não permite conclusões a respeito da relação temporal e, consequentemente, de causalidade entre a hipotensão ortostática e os fatores associados

\section{Tabela 4}

Variáveis associadas à hipotensão ortostática por subgrupo de faixa etária (menor que 44 anos, $45-59$ anos e maior que 60 anos). Estudo Longitudinal de Saúde do Adulto (ELSA-Brasil, 2008-2010).

\begin{tabular}{|c|c|c|c|c|c|c|c|c|c|}
\hline \multirow[t]{2}{*}{ Variável } & \multicolumn{3}{|c|}{$<44$ anos * } & \multicolumn{3}{|c|}{ 45-59 anos } & \multicolumn{3}{|c|}{$>60$ anos $* *$} \\
\hline & $\begin{array}{c}\text { OR } \\
\text { ajustado }\end{array}$ & IC95\% & Valor de p & $\begin{array}{c}\text { OR } \\
\text { ajustado }\end{array}$ & IC95\% & Valor de p & $\begin{array}{c}\text { OR } \\
\text { ajustado }\end{array}$ & $\mathrm{IC} 95 \%$ & Valor de $p$ \\
\hline \multicolumn{10}{|l|}{ Escolaridade } \\
\hline \multicolumn{10}{|l|}{ completo } \\
\hline Ensino Médio completo & & & & & & & 2,11 & $1,30-3,44$ & 0,003 \\
\hline Ensino Superior & & & & & & & 1,00 & & \\
\hline \multicolumn{9}{|l|}{ pulso } & \\
\hline \multicolumn{10}{|l|}{ IAM/Revascularização } \\
\hline Não & & & & - & - & - & 1,00 & & \\
\hline Sim & & & & & & & 1,88 & $1,003-3,538$ & 0,049 \\
\hline \multicolumn{10}{|l|}{ Doença cardíaca } \\
\hline Não & & & & & & & 1,00 & & \\
\hline Sim & & & & & & & 2,95 & $1,49-5,85$ & 0,002 \\
\hline \multicolumn{10}{|l|}{ Hipertensão } \\
\hline Não & & & & 1,00 & & & & & \\
\hline Sim & & & & 1,72 & $1,22-2,43$ & 0,002 & & & \\
\hline \multirow{2}{*}{\multicolumn{10}{|c|}{$\begin{array}{l}\text { Sorologia para a doença } \\
\text { de Chagas }\end{array}$}} \\
\hline & & & & & & & & & \\
\hline Não & 1,00 & & & 1,00 & & & & & \\
\hline Sim & 6,35 & $1,68-23,87$ & 0,006 & 2,26 & $0,99-5,19$ & 0,053 & & & \\
\hline \multicolumn{10}{|l|}{$\begin{array}{l}\text { Sintomas na mudança } \\
\text { postural }\end{array}$} \\
\hline Não & 1,00 & & & 1,00 & & & 1,00 & & \\
\hline Sim & 59,49 & $29,19-121,22$ & $<0,000$ & 22,92 & $14,82-35,44$ & $<0,000$ & 6,78 & $3,06-15,04$ & $<0,000$ \\
\hline
\end{tabular}

IAM: infarto agudo do miocárdio; IC95\%: intervalo de 95\% de confiança; OR: odds ratio (razão de chances).

* A variável alteração no índice tornozelo braquial foi significativa na análise bivariada nos indivíduos com menos de 44 anos, entretanto, não permaneceu no modelo final da regressão logística;

** As variáveis raça/cor, alteração no índice tornozelo braquial, pressão arterial sistólica e uso de anti-hipertensivo foram significativas nas análises bivariadas nos indivíduos com mais de 60 anos, entretanto, não permaneceram no modelo final da regressão logística. 
encontrados. Entretanto, com relação à insuficiência cardíaca e à doença coronariana, os estudos citados são coortes que apontam a hipotensão ortostática como fator de risco para esses agravos. Sobre maior PAS e hipertensão, a maioria dos estudos citados são transversais, havendo também coorte. E com relação à maior frequência ITB alterado e rigidez arterial, as análises encontradas não são longitudinais.

A associação da hipotensão ortostática com o envelhecimento, com agravos cardiovasculares (principalmente como fator de risco) e com a baixa escolaridade nos indivíduos com mais de 60 anos (robusto fator de risco socioeconômico para doenças cardiovasculares, como será discutido adiante) evidencia que a hipotensão ortostática pode ser de grande utilidade como alerta de potencial comprometimento cardiovascular, com possível agravamento futuro, como ocorreu com indivíduos nos estudos longitudinais.

A queda da pressão arterial imediata à ortostase acarreta ativação autonômica simpática para os vasos (estímulo vasoconstrictor principalmente nos membros inferiores) e coração, com aumento da força de contração e da frequência cardíaca. Caso os vasos e/ou o coração estejam com comprometimento morfológico/funcional, a resposta aos estímulos não será satisfatória e, por sua vez, não haverá aumento da resistência periférica e/ou do débito cardíaco e da pressão arterial 33. Sendo assim, a presença de hipotensão ortostática seria uma importante ferramenta de rastreamento de comprometimento cardiovascular, podendo constituir uma ferramenta importante na prevenção primária e secundária.

Sobre a doença de Chagas, não encontramos estudos que associassem a hipotensão ortostática a essa doença, provavelmente pela pouca expressividade epidemiológica dessa doença nos locais de realização dos estudos de associação (América do Norte, Europa e Ásia). Importante ressaltar que a doença de Chagas cursa com disautonomias, o que poderia explicar a forte associação encontrada, notadamente em jovens, quando os demais fatores de associação ainda não se encontram presentes. Apesar do declínio dessa doença no Brasil nas últimas décadas, ela ainda constitui, em algumas regiões, importante causa de cardiopatia crônica 34 .

Com relação à presença de sintomas após a mudança postural, essa forte associação já era esperada. No ELSA-Brasil, o relato de sintomas ocorreu em apenas 1,4\% dos indivíduos sem hipotensão ortostática, valor esse que subiu para 19,7\%, naqueles com hipotensão ortostática, e para 43\%, quando a hipotensão ortostática era definida por queda de ambas as pressões. Vale lembrar que não há informação sobre o momento exato do relato do sintoma, sendo que esse podia ocorrer imediatamente após a ortostase ou até a última aferição pressórica (cinco minutos após adoção da ortostase). Portanto, não temos dados para concluir se sintomas mais precoces se associam ou não a um desajuste autonômico mais grave. Cabe ressaltar, todavia, que a hipotensão ortostática, geralmente, ocorre sem a ocorrência de sintomas. Entretanto, parece haver mais comprometimento das funções cardiovasculares quando há sintomas, como no estudo de Alagiakrishnan et al. ${ }^{9}$, no qual a razão de taxas para insuficiência cardíaca dos sintomáticos foi de 1,57 (IC95\%: 1,16-2,11), enquanto a de todos os indivíduos com hipotensão ortostática (sintomáticos ou não) foi de 1,24 (IC95\%: 1,06-1,45). Adiciona-se ainda o aumento do risco de quedas e diminuição da mobilidade e segurança na presença de sintomas. Uma revisão sistemática sobre a eficácia dos tratamentos para hipotensão ortostática (sintomática) 35 aborda que a maioria dos ensaios sobre tratamento se concentra em indivíduos com falha autonômica. Para os indivíduos sintomáticos e que não têm falha autonômica, idosos, ou pessoas com doença vascular generalizada, é sugerida uma abordagem de investigação visando à melhoria da saúde cardiovascular.

No que diz respeito à escolaridade, inversamente associada à hipotensão ortostática, é consenso que pessoas com maior escolaridade têm menos risco de desenvolver doenças cardiovasculares 36 . Como mencionado anteriormente, a baixa escolaridade é um robusto fator de risco socioeconômico para doenças cardiovasculares; e, como tal, condiciona a exposição a situações que poderão influenciar o estado de saúde dos indivíduos, afeta o estilo de vida e interfere no acesso aos cuidados de saúde 37.

As associações identificadas no nosso estudo sugerem uma raiz não neurogênica para a hipotensão ortostática na maior parte dos indivíduos que apresentaram essa condição. No entanto, não podemos descartar a possibilidade de influência neurogênica concomitante, uma vez que não analisamos variáveis sobre a função nervosa autonômica, exceto pela variação da FC, que não mostrou associação com a hipotensão ortostática. Destaca-se que, caso existam desajustes da regulação do tono autonômico concomitante, há indícios de que eles ocorram na circulação periférica, e não no coração, uma vez que 
a resposta cronotrópica foi bastante próxima nos indivíduos com ou sem hipotensão ortostática. Vale ressaltar que, nos indivíduos sintomáticos, a hipotensão ortostática tem uma clara etiologia neurogênica em apenas uma minoria dos casos, e nenhuma causa definitiva pode ser identificada em quase $40 \%$ dos indivíduos com hipotensão ortostática moderada e grave (hipotensão ortostática idiopática) 38 . No entanto, a maioria dos pacientes com hipotensão ortostática é assintomática ou tem poucos sintomas não específicos, contribuindo com uma alta taxa de subdiagnóstico ${ }^{39}$, o que foi confirmado em nosso estudo, em que menos da metade dos portadores de hipotensão ortostática apresentou sintomas. Esse dado indica que o diagnóstico de hipotensão ortostática, mais importante em idosos como mostrado em todos os estudos, não pode ser buscado apenas investigando sintomas, e sim por meio da realização do teste ativo.

Sobre a duração do repouso anterior à ortostase, não há estudos que analisem o impacto dessa duração com os fatores relacionados à hipotensão ortostática. No presente estudo, foram empregados 20 minutos de repouso, período utilizado para a realização do ITB. Em geral, os estudos se concentram entre 5,10 e 20 minutos $8,13,16$.

Muito embora tenhamos utilizado dados de uma amostra grande, há limitações no presente estudo que merecem menção. A amostra é formada por uma classe laboral (servidores públicos). Portanto, é preciso cautela para estender os achados para outras populações ou para a população geral. Destacase, entretanto, que, na população do ELSA, parte do espectro de variação de idade, raça/cor e escolaridade existente na população brasileira está representada em ambos os sexos. Portanto, na ausência de dados populacionais, os dados do ELSA constituem, hoje, a melhor referência para a associação com hipotensão ortostática na população brasileira.

Pode-se concluir que fatores que se associaram de forma independente à presença de hipotensão ortostática foram maior faixa etária, maior PAS, alteração no ITB e presença de IAM/revascularização, doença cardíaca, sorologia positiva para a doença de Chagas e, como esperado, a ocorrência de sintomas na mudança postural. Dessa forma, a utilização do teste postural, método de identificação simples e barato da hipotensão ortostática, seria adequada para triagem. Sua utilização como rotina de exame clínico pode identificar indivíduos que necessitam de investigação da saúde cardiovascular, favorecendo o diagnóstico e o tratamento precoce de agravos e, consequentemente, a limitação de incapacidades.

\section{Colaboradores}

A. P. C. Velten trabalhou na concepção, análise e interpretação dos dados e redação do artigo. I. Benseñor trabalhou na concepção, execução da pesquisa e elaboração do artigo. J. B. Souza trabalhou na análise de dados e elaboração do artigo. J. G. Mill trabalhou na concepção do estudo, execução da pesquisa, interpretação dos dados e redação do artigo. Todos os autores aprovaram a versão final para publicação.

\section{Agradecimentos}

Ao Ministério da Saúde, Ministério da Ciência e Tecnologia e ao Conselho Nacional de Desenvolvimento Científico e Tecnológico pelo financiamento.

\section{Informações adicionais}

ORCID: Ana Paula Costa Velten (0000-0002-1176-

903X); Isabela Benseñor (0000-0002-6723-5678);

Juliana Bottoni de Souza (0000-0002-9308-7445);

José Geraldo Mill (0000-0002-0987-368X). 


\section{Referências}

1. Freeman R, Wieling W, Axelrod FB, Benditt DG, Benarroch E, Biaggioni I, et al. Consensus statement on the definition of orthostatic hypotension, neurally mediated syncope and the postural tachycardia syndrome. Clin Auton Res 2011; 21:69-72.

2. Fedorowski A, Melander O. Syndromes of orthostatic intolerance: a hidden danger. J Intern Med 2013; 273:322-35.

3. Perlmuter LC, Sarda G, Casavant V, Mosnaim AD. A review of the etiology, asssociated comorbidities, and treatment of orthostatic hypotension. Am J Ther 2013; 20:279-91.

4. Verwoert GC, Mattace-Raso FUS, Hofman A, Heeringa J, Stricker BHC, Breteler MMB, et al. Orthostatic hypotension and risk of cardiovascular disease in elderly people: the Rotterdam study. J Am Geriatr Soc 2008; 56:1816-20.

5. Agarwal SK, Alonso A, Whelton SP, Soliman EZ, Rose KM, Chamberlain AM, et al. Orthostatic change in blood pressure and incidence of atrial fibrillation: results from a bi-ethnic population based study. PLoS One 2013; 8:e79030.

6. Fedorowski A, Hedblad B, Engström G, Gustav Smith J, Melander O. Orthostatic hypotension and long-term incidence of atrial fibrillation: the Malmö preventive project. J Intern Med 2010; 268:383-9.

7. Rose KM, Holme I, Light KC, Sharrett AR, Tyroler HA, Heiss G. Association between the blood pressure response to a change in posture and the 6-year incidence of hypertension: prospective findings from the ARIC study. J Hum Hypertens 2002; 16:771-7.

8. Jones CD, Loehr L, Franceschini N, Rosamond WD, Chang PP, Shahar E, et al. Orthostatic hypotension as a risk factor for incident heart failure: the atherosclerosis risk in communities study. Hypertension 2012; 59:913-8.

9. Alagiakrishnan K, Patel K, Desai RV, Ahmed $\mathrm{MB}$, Fonarow GC, Forman DE, et al. Orthostatic hypotension and incident heart failure in community-dwelling older adults. J Gerontol A Biol Sci Med Sci 2014; 69:223-30.

10. Fedorowski A, Engström G, Hedblad B, Melander O. Orthostatic hypotension predicts incidence of heart failure: the Malmö Preventive Project. Am J Hypertens 2010; 23:1209-15.

11. Yatsuya H, Folsom AR, Alonso A, Gottesman RF, Rose KM. Postural changes in blood pressure and incidence of ischemic stroke subtypes: the ARIC study. Hypertension 2011; 57:167-73.

12. Eigenbrodt ML, Rose KM, Couper DJ, Arnett DK, Smith R, Jones D. Orthostatic hypotension as a risk factor for stroke: the Atherosclerosis Risk in Communities (ARIC) Study, 19871996. Stroke 2000; 31:2307-13.
13. Mattace-Raso FUS, van der Cammen TJM, Knetsch AM, van den Meiracker AH, Schalekamp MDH, Hofman A, et al. Arterial stiffness as the candidate underlying mechanism for postural blood pressure changes and orthostatic hypotension in older adults: the Rotterdam Study. J Hypertens 2006; 24:339-44.

14. Franceschini N, Rose KM, Brad CA, Couper DJ, Suma V. Orthostatic hypotension is associated with incident chronic kidney disease: the Atherosclerosis Risk In Communities Study. Hypertension 2010; 56:1054-9.

15. Rose KM, Eigenbrodt ML, Biga RL, Couper DJ, Light KC, Sharrett AR, et al. Orthostatic hypotension predicts mortality in middle-aged adults: the Atherosclerosis Risk in Communities (ARIC) Study. Circulation 2006; 114:6306.

16. Fedorowski A, Stavenow L, Hedblad B, Berglund G, Nilsson PM, Melander O. Orthostatic hypotension predicts all-cause mortality and coronary events in middle-aged individuals (The Malmö Preventive Project). Eur Heart J 2010; 31:85-91

17. O'Connell MDL, Savva GM, Fan CW, Kenny RA. Orthostatic hypotension, orthostatic intolerance and frailty: the Irish Longitudinal Study on Aging-TILDA. Arch Gerontol Geriatr 2015; 60:507-13.

18. Curreri C, Giantin V, Veronese N, Trevisan C, Sartori L, Musacchio E, et al. Orthostatic changes in blood pressure and cognitive status in the elderly: the Progetto Veneto Anziani Study. Hypertension 2016; 68:427-35.

19. Casiglia E, Tikhonoff V, Caffi S, Boschetti G, Giordano N, Guidotti F, et al. Orthostatic hypotension does not increase cardiovascular risk in the elderly at a population level. Am J Hypertens 2014; 27:81-8.

20. Cooke J, Carew S, Quinn C, O'Connor M, Curtin J, O'Connor C, et al. The prevalence and pathological correlates of orthostatic hypotension and its subtypes when measured using beat-to-beat technology in a sample of older adults living in the community. Age Ageing 2013; 42:709-14.

21. Rose KM, Tyroler HA, Nardo CJ, Arnett DK, Light KC, Rosamond W, et al. Orthostatic hypotension and the incidence of coronary heart disease: the Atherosclerosis Risk in Communities study. Am J Hypertens 2000; $13(6 \mathrm{Pt}$ 1):571-8

22. Wu J-S, Yang Y-C, Lu F-H, Wu C-H, Chang C-J. Population-based study on the prevalence and correlates of orthostatic hypotension/hypertension and orthostatic dizziness. Hypertens Res 2008; 31:897-904. 
23. Aquino EML, Araujo MJ, Almeida MCC, Conceição P, Andrade CR, Cade NV, et al. Participants recruitment in ELSA-Brasil (Brazilian Longitudinal Study for Adult Health). Rev Saúde Pública 2013; 47:10-8.

24. Aquino EML, Barreto SM, Bensenor IM, Carvalho MS, Chor D, Duncan BB, et al. Brazilian Longitudinal Study of Adult Health (ELSABrasil): objectives and design. Am J Epidemiol 2012; 175:315-24.

25. Schatz IJ, Bannister R, Freeman RL, Goetz CG, Jankovic J, Kaufmann HC, et al. Consensus statement on the definition of orthostatic hypotension, pure autonomic failure and multiple system atrophy. Clin Auton Res 1996; 6:125-6.

26. Mill JG, Pinto K, Griep RH, Goulart A, Foppa $\mathrm{M}$, Lotufo $\mathrm{P}$, et al. Medical assessments and measurements in ELSA-Brasil. Rev Saúde Pública 2013; 47:54-62.

27. Guidelines for Data Processing and Analysis of the International Physical Activity Questionnaire (IPAQ) - Short and Long Forms, 2005. http://www.ipaq.ki.se/scoring.pdf (acessado em 10/Dez/2017).

28. Benseñor IM, Griep RH, Pinto KA, Faria CP, Mendes MF, Caetano EI, et al. Rotinas de organização de exames e entrevistas no centro de investigação ELSA-Brasil Routines of organization of ELSA-Brasil investigation center. Rev Saúde Pública 2013; 47 Suppl 2:37-47.

29. Aquino EML, Silva PRV, Coeli CM, Araújo MJ, Santos SM, Figueiredo RC, et al. Aspectos éticos em estudos longitudinais: o caso do ELSABrasil. Rev Saúde Pública 2013; 47 Suppl 2:1926.

30. Finucane C, O'Connell MDL, Fan CW, Savva GM, Soraghan CJ, Nolan H, et al. Age-related normative changes in phasic orthostatic blood pressure in a large population study: findings from the Irish longitudinal study on ageing (TILDA). Circulation 2014; 130:1780-9.
31. Qingtao M, Si W, Yong W, Shixi W, Kai L, Zhou X, et al. Arterial stiffness is a potential mechanism and promising indicator of orthostatic hypotension in the general population. Vasa 2014; 43:423-32.

32. Vara-González L, Muñoz-Cacho P, Sanz de Castro S. Postural changes in blood pressure in the general population of Cantabria (northern Spain). Blood Press Monit 2008; 13:263-7.

33. Michelini LC. Regulação da pressão arterial: mecanismos neuro-hormonais. In: Aires MM, organizador. Fisiologia. 4a Ed. Rio de Janeiro: Guanabara Koogan; 2015. p. 571-93.

34. Dias JCP, Ramos Jr. AN, Gontijo ED, Luquetti A, Shikanai-Yasuda AM, Coura JR, et al. II Consenso Brasileiro em Doença de Chagas, 2015. Epidemiol Serv Saúde 2016; 25 n. spe:786.

35. Logan IC, Witham MD. Efficacy of treatments for orthostatic hypotension: a systematic review. Age Ageing 2012; 41:587-94.

36. Tillmann T, Vaucher J, Okbay A, Pikhart H, Peasey A, Kubinova R, et al. Education and coronary heart disease: Mendelian randomisation study. BMJ 2017; 358:j3542.

37. Sousa P. Equity, socioeconomic inequalities and cardiovascular disease. Port J Cardiol 2013; 32:855-6.

38. Robertson D, Robertson RM. Causes of chronic orthostatic hypotension. Arch Intern Med 1994; 154:1620-4.

39. Benvenuto LJ, Krakoff LR. Morbidity and mortality of orthostatic hypotension: implications for management of cardiovascular disease. Am J Hypertens 2011; 24:135-44. 


\section{Abstract}

This study aimed to investigate factors associated with orthostatic hypotension in 14,833 individuals 35-74 years of age. This was a cross-sectional study of baseline data (2008-2010) from the Longitudinal Study of Adult Health (ELSA-Brasil). Postural testing was performed after 20 minutes resting in supine position and active adoption of orthostatic posture. Blood pressure was measured in supine position and at 3 minutes in orthostatic position with an oscillometer (HEM $705 \mathrm{CP}$, Omron, São Paulo, Brazil). Orthostatic hypotension was defined as a drop of $\geq 20 \mathrm{mmHg}$ in systolic blood pressure and/or a drop of $\geq 10 \mathrm{mmHg}$ in diastolic blood pressure. The target covariates were sex, age bracket, race/color, schooling, nutritional status, waist circumference, alteration in the ankle-brachial index, pulse wave velocity, systolic and diastolic blood pressure, hypertension, diabetes, use of antihypertensives, cholesterol, triglycerides, Chagas disease serology, symptoms, and heart rate variation in the postural test, selfreported heart disease, acute myocardial infarction $(A M I) /$ revascularization, and stroke. Orthostatic hypotension was significantly associated with higher age bracket, $O R=1.83$ (95\%CI: 1.14-2.95); alteration in the ankle-brachial index, $O R=$ 2.8 (95\%CI: 1.13-6.88); AMI/revascularization, $O R=1.70$ (95\%CI: 1.01-2.87); report of heart disease, $O R=3.03$ (95\%CI: 1.71-5.36); increased systolic blood pressure, OR $=1.012$ (95\%CI: 1.006-1.019); positive Chagas disease serology, $O R=2.29$ (95\%CI: 1.23-4.27); and occurrence of symptoms with postural change, $O R=20.81$ (95\%CI: 14.81-29.24). Presence of orthostatic hypotension can be a warning sign for cardiovascular disorders and thus a useful tool for screening and prevention.

Orthostatic Hypotension; Cardiovascular

Diseases; Adult Health

\section{Resumen}

El objetivo fue investigar los factores asociados a la presencia de hipotensión ortostática en 14.833 individuos de 35-74 años. Se realizó un estudio transversal con los datos de la línea de base (20082010) del Estudio Longitudinal de Salud del Adulto (ELSA-Brasil). El examen postural se realizó tras un reposo de 20 minutos en posición supina y la adopción activa de la postura ortostática. Se midió la presión arterial en supino y a los 3 minutos de ortostasis con aparato oscilométrico (HEM 705 CP, Omron, São Paulo, Brasil). La hipotensión ortostática se definió por la caí$d a \geq 20 \mathrm{mmHg}$ en la presión arterial sistólica $y / o$ caída $\geq 10 \mathrm{mmHg}$ en la presión arterial diastólica. Las covariables analizadas fueron sexo, franja de edad, raza/color, escolaridad, estado nutricional, circunferencia de la cintura, alteración en el indice tobillo-brazo, velocidad de onda de pulso, presión arterial sistólica y diastólica, hipertensión, diabetes, uso de antihipertensivos, colesterol, triglicéridos, serología para a enfermedad de Chagas, ocurrencia de sintomas y variación de frecuencia cardíaca en el examen postural, informe de enfermedad cardíaca, infarto agudo de miocardio (IAM)/revascularización y accidente vascular cerebral. La hipotensión ortostática estuvo significativamente asociada a la mayor franja de edad, $O R=1,83$ (IC95\%: 1,14-2,95); alteración en el indice tobillo-brazo, $O R=2,8$ (IC95\%: 1,13-6,88), $I A M /$ revascularización, $O R=1,70$ (IC95\%: 1,01 2,87); relato de enfermedad cardíaca, $O R=3,03$ (IC95\%: 1,71-5,36); presión arterial sistólica aumentada, OR = 1,012 (IC95\%: 1,006-1,019); serología positiva para a enfermedad de Chagas, $O R=$ 2,29 (IC95\%: 1,23-4,27) y ocurrencia de sintomas en el cambio postural, OR = 20,81 (IC95\%: 14,81-29,24). La presencia de hipotensión ortostática puede ser una alerta de potencial comprometimiento cardiovascular, $y$, por tanto una herramienta de seguimiento y prevención.

Hipotensión Ortostática; Enfermedades

Cardiovasculares; Salud del Adulto
Recebido em 22/Jun/2018

Versão final reapresentada em 07/Mar/2019 Aprovado em 15/Mar/2019 